\title{
CARLEMAN'S FORMULA OF A SOLUTIONS OF THE POISSON EQUATION IN BOUNDED DOMAIN
}

\author{
Ermamat N. Sattorov ${ }^{\dagger}$, Zuxro E. Ermamatova ${ }^{\dagger \dagger}$ \\ Samarkand State University, \\ Samarkand boulevard 15, Samarkand, Uzbekistan \\ †Sattorov-e@rambler.ru, †† zuxroermamatova@rambler.ru
}

\begin{abstract}
We suggest an explicit continuation formula for a solution to the Cauchy problem for the Poisson equation in a domain from its values and values of its normal derivative on a part of the boundary. We construct the continuation formula of this problem based on the Carleman-Yarmuhamedov function method.

Keywords: Poisson equations, Ill-posed problem, Regular solution, Carleman-Yarmuhamedov function, Green's formula, Carleman formula, Mittag-Leffler entire function.
\end{abstract}

\section{Introduction}

In this paper, we continue the research provided in [12]. We propose an explicit formula for the reconstruction of a solution of the Poisson equation in a bounded domain from its values and the values of its normal derivative on a part of the boundary, i.e., we give an explicit continuation formula for a solution to the Cauchy problem for the Poisson equation.

Let us introduce the following notation: $\mathbb{R}^{3}$ is a three-dimensional real Euclidean space,

$$
\begin{gathered}
x=\left(x_{1}, x_{2}, x_{3}\right), \quad y=\left(y_{1}, y_{2}, y_{3}\right) \in \mathbb{R}^{3}, \\
x^{\prime}=\left(x_{1}, x_{2}\right), \quad y^{\prime}=\left(y_{1}, y_{2}\right) \in \mathbb{R}^{2}, \\
s=\alpha^{2}=\left|y^{\prime}-x^{\prime}\right|^{2}=\left(y_{1}-x_{1}\right)^{2}+\left(y_{2}-x_{2}\right)^{2}, \\
r^{2}=s+\left(y_{3}-x_{3}\right)^{2}=|y-x|^{2}, \quad \tau=\operatorname{tg} \frac{\pi}{2 \rho}, \quad \rho>1, \\
G_{\rho}=\left\{y:\left|y^{\prime}\right|<\tau y_{3}, \quad y_{3}>0\right\}, \quad \partial G_{\rho}=\left\{y:\left|y^{\prime}\right|=\tau y_{3}, \quad y_{3}>0\right\}, \quad \bar{G}_{\rho}=G_{\rho} \cup \partial G_{\rho},
\end{gathered}
$$

$\varepsilon, \varepsilon_{1}$, and $\varepsilon_{2}$ are sufficiently small positive constants,

$$
G_{\rho}^{\varepsilon}=\left\{y:\left|y^{\prime}\right|<\tau\left(y_{3}-\varepsilon\right)\right\}, \quad \partial G_{\rho}^{\varepsilon}=\left\{y:\left|y^{\prime}\right|=\tau\left(y_{3}-\varepsilon\right)\right\}, \quad \bar{G}_{\rho}^{\varepsilon}=G_{\rho}^{\varepsilon} \cup \partial G_{\rho}^{\varepsilon},
$$

and $\Omega_{\rho}$ is a bounded simply connected domain whose boundary $\partial \Omega_{\rho}$ in $R^{3}$ consists of a part of the conic surface $T \equiv \partial G_{\rho}$ and a smooth surface $S$ lying inside the cone $\bar{G}_{\rho}$. The case $\rho=1$ is the limit case. In this case, $G_{1}$ is the half-space $y_{3}>0, \partial G_{1}$ is the hyperplane $y_{3}=0$, and $\Omega_{1}$ is a bounded simply connected domain whose boundary consists of a compact connected part of the hyperplane $y_{3}=0$ and a smooth surface $S$ in the half-space $y_{3} \geq 0, \bar{\Omega}_{\rho}=\Omega_{\rho} \cup \partial \Omega_{\rho}$, and $S_{0}$ is the interior of $S$.

The Poisson equation or potential equation [15]

$$
-\triangle U(x) \equiv-\sum_{i=1}^{3} \frac{\partial^{2} U}{\partial x_{i}^{2}}=f(x)
$$

is a classical example of second-order elliptic partial differential equations and a mathematical model for some important physical phenomena. Let $H^{\lambda}\left(\Omega_{\rho}\right)$ be the set of real functions of the class 
$C^{2, \lambda}\left(\Omega_{\rho}\right) \cap C^{1}\left(\bar{\Omega}_{\rho}\right)$ satisfying the Poisson equation. Let a function $f$ be Hölder continuous with exponent $\lambda \in(0,1)$, i.e., $f \in C^{s, \lambda}\left(\bar{\Omega}_{\rho}\right)$ and $s \in Z_{+}$.

Problem 1. Assume that we know the Cauchy data for a solution to equation (1.1) on the surface $S$ :

$$
U(y)=f_{1}(y), \quad \frac{\partial U(y)}{\partial n}=f_{2}(y), \quad y \in S
$$

where $n=\left(n_{1}, n_{2}, n_{3}\right)$ is the outward unit normal to the surface $\partial \Omega_{\rho}$ at a point $y$, and $f_{1}$ and $f_{2}$ are continuous functions. Given $f_{1}(y)$ and $f_{2}(y)$ on $S$, find $U(x), x \in \Omega_{\rho}$.

Problem 2. Let $f_{1}$ and $f_{2}$ be given on $S$. Find conditions on $f_{1}$ and $f_{2}$ that are necessary and sufficient for the existence of a solution to system (1.1) satisfying (1.2) and from the class $H\left(\Omega_{\rho}\right)$.

It is well-known that the Cauchy problem (1.2) for the Poisson equation (1.1) is ill-posed [3, 5]. Hadamard [17] noted that a solution to Problem 1 is not stable. The possibility of introducing a positive parameter $\sigma$, depending on the accuracy of the initial data, was noticed by M.M. Lavrentev [23]. The uniqueness of the solution follows from the general theorem by Holmgren [6]. It has applications in many different areas such as plasma physic, electrocardiography, and corrosion non-destructive evaluation (e.g., [7, 9, 10, 13, 19]). Traditionally, regularization techniques, such as Tikhonov regularization [44] and the quasi-reversibility approach [22], were used to provide robust numerical schemes [18].

We suppose that a solution to the problem exists (in this event, it is unique) and is continuously differentiable in the closed domain, and the Cauchy data are given exactly. In this case, we establish an explicit continuation formula. This formula enables us to state a simple and convenient criterion for the solvability of the Cauchy problem.

The result established here is a multidimensional analog of theorems and Carleman-type formulas [4] by G.M. Goluzin, V.I. Krylov, V.A. Fok, and F.M. Kuni in the theory of holomorphic functions of one variable $[14,16]$.

The method for obtaining these results is based on an explicit form of the fundamental solution of the Poisson equation which depends on a positive parameter that vanishes together with its derivatives on a fixed cone and outside it, as the parameter tends to infinity, while the pole of the fundamental solution lies inside the cone. Following to M.M. Lavrent'ev, a fundamental solution with these properties is called a Carleman function for the cone [8, 23]. Having constructed a Carleman function explicitly, we write a continuation formula. The existence of a Carleman function follows from S.N. Mergelyan's approximation theorem [28]. However, this theorem shows no way for writing the Carleman function explicitly.

The Carleman function of the Cauchy problem for the Laplace equation and some close problems, in the case when $\partial \Omega_{\rho} \backslash S$ is a part of a conic surface, was constructed in [45]. Mergelyan [28] suggested a method to construct the Carleman function of the Cauchy problem for the Laplace equation in the case when $S$ is a part, with a smooth boundary, of the boundary of a simplyconnected domain. Based on [28] and approximative theorems, the Carleman matrix for elliptic systems was constructed in [41].

In [1], some theorems of existence of the Carleman matrix and a solvability criterion for a wider class of boundary value problems for elliptic systems were established. It was proved earlier in $[1,41]$ that, for every Cauchy problem for elliptic systems, the Carleman matrix exists if the Cauchy data are given on a boundary set of positive measure.

Following Tikhonov [21, 43], we call the family of functions $U_{\sigma \delta}(x)$ the regularized solution to the Cauchy problem for equation (1.1). The regularized solution determines the stability of the approximate method. 
In the paper, based on results from $[23,45-48]$ on the Cauchy problem for the Laplace and Helmholtz equations, we construct the Carleman-Yarmuhamedov function in an explicit form. We use it to prove the Carleman formulas and a criterion for the solvability of the Cauchy problem.

In recent decades, interest in the classical ill-posed problems of mathematical physics has been preserved. This direction of investigation of the properties of solutions to the Cauchy problem for the Laplace equation was started in $[2,20,23,24,42]$ and was further developed in [25-27, 30-40].

\section{Construction of a Carleman-Yarmukhamedov function}

According to [45], we define the Carleman-Yarmukhamedov function $\Phi(y, x)$ by the equality

$$
-2 \pi^{2} K(0) \Phi(y, x)=\int_{0}^{\infty} \operatorname{Im}\left[\frac{K(w)}{w}\right] \frac{d u}{\sqrt{s+u^{2}}}, \quad w=i \sqrt{s+u^{2}}+y_{3}-x_{3} .
$$

Here, $K(w)$ is an entire function of complex variable that takes real values for real $w(w=a+i b$, $a$ and $b$ are real numbers) such that $K(a) \neq \infty,|a|<\infty, K(0) \neq 0, \forall R>0, \exists C_{R}>0$

$$
\sup _{|\operatorname{Re} w|<R, \operatorname{Im} w \leq-C_{R}}\left(|K(w)|+|\operatorname{Im} w|\left|K^{\prime}(w)\right|+|\operatorname{Im} w|^{2}\left|K^{\prime \prime}(w)\right|\right)<\infty .
$$

For real $w$, since $K(w)$ is real, we have $\overline{K(\bar{w})}=K(w)$. Then (2.1) implies that $\forall R>0$

$$
\sup _{|\operatorname{Re} w|<R}\left\{|K(w)|+(1+|\operatorname{Im} w|)\left|K^{\prime}(w)\right|+\left(1+|\operatorname{Im} w|^{2}\right)\left|K^{\prime \prime}(w)\right|\right\}<\infty .
$$

Now we write (2.1) in the form

$$
-2 \pi^{2} K(0) \Phi(y, x)=\int_{0}^{\infty}\left\{\frac{\left(y_{3}-x_{3}\right) \operatorname{Im} K(w)}{\sqrt{s+u^{2}}}-\operatorname{Re} K(w)\right\} \frac{d u}{r^{2}+u^{2}},
$$

where

$$
\begin{gathered}
\operatorname{Im}\left(\frac{K(w)}{w}\right)=\frac{1}{2 i}\left\{\frac{K(w)}{w}-\frac{K(\bar{w})}{\bar{w}}\right\}=\frac{\bar{w} K(w)-w K(\bar{w})}{2 i\left(r^{2}+u^{2}\right)} \\
=\frac{\left(y_{3}-x_{3}\right) \operatorname{Im} K(w)-\sqrt{s+u^{2}} \operatorname{Re} K(w)}{r^{2}+u^{2}} .
\end{gathered}
$$

From (2.2) and (2.3), it follows that, for $y \neq x$, the integral in (2.1) converges absolutely.

If $K(w) \equiv 1$, then the function $\Phi(y, x)$ is the classical fundamental solution to the Laplace equation, i.e.,

$$
\Phi(y, x) \equiv \Phi_{0}(r)=1 /(4 \pi r)
$$

Theorem 1 [45]. The function $\Phi(y, x)$ defined by (2.1) or (2.3)-(2.4) is representable in the form

$$
\Phi(y, x)=\Phi_{0}(r)+G(y, x),
$$

where $\Phi_{0}(r)=1 /(4 \pi r)$ and the function $G(y, x)$ is harmonic in the variable $y$ in $\mathbb{R}^{3}$, including $y=x$. 
From Theorem 1 it follows that the function $\Phi(y, x)$ of the variable $y$ is a fundamental solution of the Poisson equation. Therefore, for the function $U(y) \in H\left(\Omega_{\rho}\right)$ and for every point $x \in \Omega_{\rho}$, the Green's formula is valid [15]:

$$
U(x)=\int_{\Omega_{\rho}} \Phi(y, x) f(y) d y-\int_{\partial \Omega_{\rho}}\left[U(y) \frac{\partial \Phi(y, x)}{\partial n}-\Phi(y, x) \frac{\partial U(y)}{\partial n}\right] d S_{y},
$$

where $f(x) \in C^{\lambda}\left(\Omega_{\rho}\right), \lambda \in(0,1)$, is bounded, i.e., the former integral on the right-hand side of (2.5) satisfies equation (1.1) in the domain.

\section{The Mittag-Leffler entire function}

The continuation formulas below are expressed explicitly in terms of the Mittag-Leffler entire function; therefore, we now present its basic properties without proof. These properties as well as detailed proofs can be found in [11, Chapter 3, §2], [47].

The Mittag-Leffler entire function is defined by the series

$$
E_{\rho}(w)=\sum_{n=0}^{\infty} \frac{w^{n}}{\Gamma(1+n / \rho)}, \quad \rho>0, \quad w \in C, \quad E_{1}(w)=e^{w},
$$

where $\Gamma$ is the Euler gamma-function. Hereinafter, we suppose that $\rho>1$. Let

$$
\gamma=\gamma(1, \beta), \quad 0<\beta<\frac{\pi}{\rho}, \quad \rho>1,
$$

be the contour in the complex $w$-plane that consists of the ray $\arg w=-\beta,|w| \geq 1$, the $\operatorname{arc}$ $-\beta \leq \arg w \leq \beta$ of the circle $|w|=1$, and the ray $\arg w=\beta,|w| \geq 1$, which is passed so that $\arg w$ does not decrease. The contour $\gamma$ splits the complex domain $C$ into the two simply connected infinite domains $\Omega^{-}$and $\Omega^{+}$lying to the left and to the right of $\gamma$, respectively. We suppose that

$$
\frac{\pi}{2 \rho}<\beta<\frac{\pi}{\rho}, \quad \rho>1 .
$$

Under these conditions, the following integral representations are valid:

$$
\begin{gathered}
E_{\rho}(w)=\rho e^{w^{\rho}}+\psi_{\rho}(w), \quad w \in \Omega^{+}, \\
E_{\rho}(w)=\psi_{\rho}(w), \quad E_{\rho}^{\prime}(w)=\psi_{\rho}^{\prime}(w), \quad w \in \Omega^{-},
\end{gathered}
$$

where

$$
\psi_{\rho}(w)=\frac{\rho}{2 \pi i} \int_{\gamma} \frac{e^{\zeta^{\rho}}}{\zeta-w} d \zeta, \quad \psi_{\rho}^{\prime}(w)=\frac{\rho}{2 \pi i} \int_{\gamma} \frac{e^{\zeta^{\rho}}}{(\zeta-w)^{2}} d \zeta .
$$

Since $E_{\rho}(w)$ takes real vales for real $w$, we obtain

$$
\begin{gathered}
\operatorname{Re} \psi_{\rho}(w)=\frac{\psi_{\rho}(w)+\psi_{\rho}(\bar{w})}{2}=\frac{\rho}{2 \pi i} \int_{\gamma} \frac{e^{\zeta^{\rho}}(\zeta-\operatorname{Re} w)}{(\zeta-w)(\zeta-\bar{w})} d \zeta \\
\operatorname{Im} \psi_{\rho}(w)=\frac{\psi_{\rho}(w)-\psi_{\rho}(\bar{w})}{2 i}=\frac{\rho \operatorname{Im} w}{2 \pi i} \int_{\gamma} \frac{e^{\zeta^{\rho}}}{(\zeta-w)(\zeta-\bar{w})} d \zeta \\
\operatorname{Im} \frac{\psi_{\rho}^{\prime}(w)}{\operatorname{Im} w}=\frac{\rho}{2 \pi i} \int_{\gamma} \frac{2 e^{\zeta^{\rho}}(\zeta-\operatorname{Re} w)}{(\zeta-w)^{2}(\zeta-\bar{w})^{2}} d \zeta
\end{gathered}
$$


Hereinafter, we take

$$
\beta=\frac{\pi}{2 \rho}+\frac{\varepsilon_{2}}{2}, \quad \rho>1,
$$

in the definition of the contour $\gamma(1, \beta)$. It is clear that, if

$$
\frac{\pi}{2 \rho}+\varepsilon_{2} \leq|\arg w| \leq \pi
$$

then $w \in \Omega_{\rho}^{-}$and $E_{\rho}(w)=\psi_{\rho}(w)$.

Define

$$
T_{k, p}(w)=\frac{\rho}{2 \pi i} \int_{\gamma} \frac{\zeta^{p} e^{\zeta^{p}}}{(\zeta-w)^{k}(\zeta-\bar{w})^{k}} d \zeta, \quad k=1,2, \ldots, \quad p=0,1, \ldots
$$

The following inequalities are valid for $\pi /(2 \rho)+\varepsilon_{2} \leq|\arg w| \leq \pi$ :

$$
\begin{gathered}
\left|E_{\rho}(w)\right| \leq \frac{C_{1}}{1+|w|}, \quad\left|E_{\rho}^{\prime}(w)\right| \leq \frac{C_{2}}{1+|w|^{2}}, \\
\left|T_{k, p}(w)\right| \leq \frac{C_{3}}{1+|w|^{2 k}}, \quad k=1,2, \cdots,
\end{gathered}
$$

where $C_{1}, C_{2}$, and $C_{3}$ are constants independent of $w$. Take in (2.1)

$$
\beta=\frac{\pi}{2 \rho}+\frac{\varepsilon_{2}}{2}<\frac{\pi}{\rho}, \quad \rho>1 .
$$

Then $E_{\rho}(w)=\psi_{\rho}(w)$, where $\psi_{\rho}(w)$ is defined by (3.1). Moreover, note that $\cos \rho \beta<0$ and the integral converges:

$$
\int_{\gamma}|\zeta|^{p} e^{\cos \rho \beta|\zeta|^{\rho}}|d \zeta|<\infty, \quad p=0,1, \ldots
$$

\section{Carleman formulas}

Let the Mittag-Leffler entire function be the function $K(w)$ in $(2.1)$ :

$$
K(w)=e^{a w^{2}} E_{\rho}(\sigma w),
$$

where

$$
\rho>1, \quad w=i \sqrt{s+u^{2}}+y_{3}-x_{3}, \quad K(0)=E_{\rho}(0)=1, \quad a>0 \quad \sigma \geq 0 .
$$

Denote by $\Phi_{\sigma}(y, x)$ the corresponding fundamental solution and by $\Phi_{\sigma}(y-x)$ its derivative with respect to the variable $\sigma$ :

$$
\Psi_{\sigma}(y-x) \equiv \frac{d \Phi_{\sigma}}{d \sigma}(y-x)
$$

It follows from Theorem 1 that $\Psi_{\sigma}(y-x)$ satisfies the Poisson equation in $\mathbb{R}^{3}$. Then

$$
\begin{gathered}
-2 \pi^{2} \Phi_{\sigma}(y-x)=\int_{0}^{\infty} \operatorname{Im}\left[\frac{e^{a w^{2}} E_{\rho}(\sigma w)}{w}\right] \frac{d u}{\sqrt{s+u^{2}}} \\
=e^{a\left(y_{3}-x_{3}\right)^{2}} \int_{0}^{\infty} \varphi_{\sigma}(y, x, u) \frac{e^{-a s-a u^{2}}}{u^{2}+r^{2}} d u,
\end{gathered}
$$


where

$$
\begin{gathered}
\varphi_{\sigma}(y-x, u)=\left[\frac{\left(y_{3}-x_{3}\right)}{\sqrt{u^{2}+s}} \operatorname{Im} E_{\rho}(\sigma w)-\operatorname{Re} E_{\rho}(\sigma w)\right] \cos \left(\nu \sqrt{s+u^{2}}\right) \\
+\left[\operatorname{Im} E_{\rho}(\sigma w)+\frac{\left(y_{3}-x_{3}\right)}{\sqrt{s+u^{2}}} \operatorname{Re} E_{\rho}(\sigma w)\right] \sin \left(\nu \sqrt{s+u^{2}}\right), \quad \nu=2 a\left(y_{3}-x_{3}\right), \\
\Psi_{\sigma}(y-x) \equiv \frac{d \Phi_{\sigma}}{d \sigma}(y-x)=\int_{0}^{\infty} \operatorname{Im}\left[e^{a w^{2}} E_{\rho}^{\prime}(\sigma w)\right] \frac{d u}{\sqrt{s+u^{2}}} .
\end{gathered}
$$

Lemma 1 [47]. Let $M$ be a compact set in $G_{\rho}$, and let $\delta$ be the distance from $M$ to $\partial G_{\rho}$. Then, for $\sigma \geq 0$, the following inequalities are valid for $x \in M$ and $y \in R^{3} \backslash G_{\rho}\left(\left|y^{\prime}\right| \geq \tau y_{3}\right)$ :

$$
\begin{gathered}
\left|\Phi_{\sigma}(y-x)\right|+\left|\frac{\partial}{\partial y_{k}} \Phi_{\sigma}(y-x)\right|+\left|\frac{\partial^{i}}{\partial x_{j}^{i}} \frac{\partial}{\partial y_{k}} \Phi_{\sigma}(y-x)\right| \leq \frac{C_{4}(\rho, \delta) r}{1+\sigma \delta}, \\
\quad r \geq \delta>0, \quad i,=0,1, \quad k, j=1,2,3 . \\
\left|\Psi_{\sigma}(y-x)\right|+\left|\frac{\partial}{\partial y_{k}} \Psi_{\sigma}(y-x)\right|+\left|\frac{\partial^{i}}{\partial x_{j}^{i}} \frac{\partial}{\partial y_{k}} \Psi_{\sigma}(y-x)\right| \leq \frac{C_{5}(\rho, \delta) r}{1+\sigma \delta}, \\
\quad r \geq \delta>0, \quad i=0,1, \quad k, j=1,2,3,
\end{gathered}
$$

where the constants $C_{4}$ and $C_{5}$ are independent of $x, y$, and $\sigma$.

Theorem 2. Let $f$ be bounded and locally Hölder continuous in $\Omega_{\rho}, U(y) \in H^{\lambda}\left(\Omega_{\rho}\right)$, and

$$
U(y)=f_{1}(y), \quad \frac{\partial U}{\partial n}(y)=f_{2}(y), \quad y \in S,
$$

where $f_{1}(y)$ and $f_{2}(y)$ are given functions of the class $C(S)$. Then the Carleman formulas

$$
\begin{gathered}
\frac{\partial^{i} U(x)}{\partial x_{j}^{i}}=\lim _{\sigma \rightarrow \infty} \frac{\partial^{i} U_{\sigma}(x)}{\partial x_{j}^{i}} \\
=\lim _{\sigma \rightarrow \infty}\left[\int_{\Omega_{\rho}} f(y) \frac{\partial^{i} \Phi_{\sigma}(y-x)}{\partial x_{j}^{i}} d y-\int_{S}\left\{f_{1}(y) \frac{\partial^{i}}{\partial x_{j}^{i}} \frac{\partial \Phi_{\sigma}(y-x)}{\partial n}-f_{2}(y) \frac{\partial^{i} \Phi_{\sigma}(y-x)}{\partial x_{j}^{i}}\right\} d S_{y}\right]
\end{gathered}
$$

are valid for every $x \in \Omega_{\rho}$, where $i=0,1, j=1,2,3$,

$$
\frac{\partial^{0} U_{\sigma}}{\partial x_{j}^{0}}=U_{\sigma}, \quad \frac{\partial^{0} \Phi_{\sigma}}{\partial x_{j}^{0}}=\Phi_{\sigma}
$$

and the convergence in (4.5) is uniform on compact sets in $\Omega_{\rho}$.

P r o o f. From Green's formula (2.5), for every $x \in \Omega_{\rho}$, we obtain

$$
\frac{\partial^{i} U(x)}{\partial x_{j}^{i}}=\int_{\Omega_{\rho}} f(y) \frac{\partial^{i} \Phi_{\sigma}(y-x)}{\partial x_{j}^{i}} d y-\int_{\partial \Omega_{\rho}}\left[f_{1}(y) \frac{\partial^{i}}{\partial x_{j}^{i}} \frac{\partial \Phi_{\sigma}(y-x)}{\partial n}-f_{2}(y) \frac{\partial^{i}}{\partial x_{j}^{i}} \Phi_{\sigma}(y-x)\right] d S_{y},
$$

$\partial \Omega_{\rho}=S \cup\left(\partial \Omega_{\rho} \backslash S\right)$. According to [47], let us estimate

$$
\Phi_{\sigma}, \quad \frac{\partial \Phi_{\sigma}}{\partial y_{j}}, \quad \frac{\partial^{i}}{\partial x_{j}^{i}} \frac{\partial \Phi_{\sigma}}{\partial y_{j}} .
$$


Lemma 1 yields the assertion of Theorem 2. Indeed, if $M$ is a compact set in $\Omega_{\rho}$ then $M \subset G_{\rho}$. Therefore, the inequalities in Lemma 1 for $\Phi_{\sigma}(y-x)$ and its derivatives remain also valid in the case where $x \in M \subset \Omega_{\rho}$ and $y \in \partial \Omega_{\rho} \backslash S \subset \partial G_{\rho}$ (in this case, $\delta$ is the distance from the compact set $M \subset \Omega_{\rho}$ to $\partial \Omega_{\rho}$ ). Now, let $\sigma$ tend to infinity. The proof of Theorem 2 is complete.

We can write (4.5) in the following equivalent form:

$$
\begin{gathered}
\frac{\partial^{i} U(x)}{\partial x_{j}^{i}}=\int_{0}^{\infty} \frac{\partial^{i}}{\partial x_{j}^{i}} J(\sigma, x)+\int_{\Omega_{\rho}} f(y) \frac{\partial^{i} \Phi_{0}(r)}{\partial x_{j}^{i}} d y \\
-\int_{S}\left[f_{1}(y) \frac{\partial^{i}}{\partial x_{j}^{i}} \frac{\partial \Phi_{0}(r)}{\partial n}-f_{2}(y) \frac{\partial^{i} \Phi_{0}(r)}{\partial x_{j}^{i}}\right] d S_{y}, \quad x \in \Omega_{\rho},
\end{gathered}
$$

where

$$
\begin{gathered}
\frac{\partial^{i}}{\partial x_{j}^{i}} J(\sigma, x)=\int_{\Omega_{\rho}} f(y) \frac{\partial^{i} \Psi_{\sigma}(y-x)}{\partial x_{j}^{i}} d y-\int_{S}\left[f_{1}(y) \frac{\partial^{i}}{\partial x_{j}^{i}} \frac{\partial \Psi_{\sigma}(y-x)}{\partial n}-f_{2}(y) \frac{\partial^{i} \Psi_{\sigma}(y-x)}{\partial x_{j}^{i}}\right] d S_{y}, \\
x \in \Omega_{\rho}, \quad i=0,1, \quad j=1,2,3, \quad \frac{\partial^{0} U}{\partial x_{j}^{0}}=U, \quad \frac{\partial^{0} \Phi_{0}}{\partial x_{j}^{0}}=\Phi_{0}, \quad \frac{\partial^{0} \Psi_{\sigma}}{\partial x_{j}^{0}}=\Psi_{\sigma}, \quad \frac{\partial^{0} J}{\partial x_{j}^{0}}=J .
\end{gathered}
$$

The functions $\Psi_{\sigma}(y-x)$ and $\Phi_{0}(r)$ are defined by equalities (4.2) and (4.1), respectively. The proof of (4.7) follows from the formulas

$$
\lim _{\sigma \longrightarrow \infty} \frac{\partial^{i}}{\partial x_{j}^{i}} P(\sigma, x)=\int_{0}^{\infty} \frac{\partial^{i}}{\partial x_{j}^{i}} \frac{\partial P(\sigma, x)}{\partial \sigma}+\frac{\partial^{i}}{\partial x_{j}^{i}} P(x)
$$

and

$$
\begin{gathered}
\frac{\partial^{i}}{\partial x_{j}^{i}} \frac{\partial P(\sigma, x)}{\partial \sigma}=\int_{\Omega_{\rho}} f(y) \frac{\partial^{i} \Psi_{\sigma}(y-x)}{\partial x_{j}^{i}} d y-\int_{S}\left[f_{1}(y) \frac{\partial^{i}}{\partial x_{j}^{i}} \frac{\partial \Psi_{\sigma}}{\partial n}(y-x)-f_{2}(y) \frac{\partial^{i} \Psi_{\sigma}}{\partial x_{j}^{i}}(y-x)\right] d S_{y}, \\
x \in \Omega_{\rho}, \quad i=0,1, \quad j=1,2,3 ;
\end{gathered}
$$

moreover, the differentiation under the integral sign is legal and

$$
\frac{\partial^{i}}{\partial x_{j}^{i}} \frac{\partial P(\sigma, x)}{\partial \sigma}=\frac{\partial^{i}}{\partial x_{j}^{i}} J(\sigma, x) .
$$

Theorem 3. Let $S \subset C^{2}, f_{1}(y) \in C^{1}\left(S_{0}\right) \cap L(S), f_{2}(y) \in C\left(S_{0}\right) \cap L(S)$, and let $f$ be bounded and locally Hölder continuous in $\Omega_{\rho}$. Then for the existence of a function $U(y) \in H^{\lambda}\left(\Omega_{\rho}\right) \cap C\left(S_{0}\right)$ such that

$$
U(y)=f_{1}(y), \quad \frac{\partial U}{\partial n}(y)=f_{2}(y), \quad y \in S_{0},
$$

it is necessary and sufficient that the following improper integral converge (uniformly on compact sets in $G_{\rho}$ ) for each $x \in G_{\rho}$ :

$$
\left|\int_{1}^{\infty} J(\sigma, x) d \sigma\right|<\infty
$$

where $J(\sigma, x)$ is defined by (4.8). If (4.10) is satisfied, then harmonic continuation is performed by equivalent formulas (4.5) and (4.7). 
P r o o f. Necessity: Let

$$
U(y) \in H\left(\Omega_{\rho}\right) \cap C^{1}\left(\Omega_{\rho} \cup S_{0}\right) \cap L(S)
$$

satisfy (4.10). Let $M$ be a compact set in $G_{\rho}$, and let $\varepsilon>0$ be such that $M \subset \bar{G}_{\rho}^{2 \varepsilon} \subset \bar{G}_{\rho}^{\varepsilon} \subset G_{\rho}$. It is clear that the distance from $M$ to $\partial G_{\rho}^{\varepsilon}$ is at least $\varepsilon \tau_{1}$ and the distance from $\partial G_{\rho}^{2 \varepsilon}$ to $\partial G_{\rho}^{\varepsilon}$ is $\varepsilon \tau_{1}$. Now, let $y \in R^{3} \backslash G_{\rho}^{\varepsilon}\left(\left|y^{\prime}\right| \leq \tau\left(y_{3}-\varepsilon\right)\right.$ and $\left.y_{3}>\varepsilon\right)$ and $x \in M\left(\left|x^{\prime}\right| \leq \tau\left(x_{3}-2 \varepsilon\right)\right.$ and $\left.x_{3}>2 \varepsilon\right)$. Then $\arg w=\arg (\sigma w)=\arg \left(i \tau \sqrt{u^{2}+s}+\tau y_{3}-\tau x_{3}\right)$ and

$$
\begin{aligned}
& \tau w=i \tau \sqrt{u^{2}+s}+\tau y_{3}-\tau x_{3}=\sqrt{u^{2}+s}\left(i \operatorname{tg} \frac{\pi}{2 \rho}+\frac{\tau y_{3}-\tau x_{3}}{\sqrt{u^{2}+s}}\right), \quad u \geq 0, \quad \rho>1, \\
& \frac{\tau y_{3}-\tau x_{3}}{\sqrt{u^{2}+s}} \leq \frac{\left|y^{\prime}\right|-\left|x^{\prime}\right|-\varepsilon \tau}{\left|y^{\prime}-x^{\prime}\right|} \leq 1-\varepsilon_{1}, \quad y^{\prime} \neq x^{\prime}, \quad\left|\arg \left(a \pm \operatorname{tg} \frac{\pi}{2 \rho}\right)\right| \geq \frac{\pi}{2 \rho} ; \quad a \leq 1 .
\end{aligned}
$$

Therefore, (2.5) is valid for $\arg w$; moreover, if $y^{\prime}=x^{\prime}$, then $\operatorname{Re} w<0$, and this inequality also holds. Consequently, $\Phi_{\sigma}(y-x)$ and $\Psi_{\sigma}(y-x)$ satisfy estimates (3.2)-(3.5) from Lemma 1, where $\delta \geq \varepsilon \tau_{1}$. Define $S_{\varepsilon}=\bar{G}_{\rho}^{\varepsilon} \cap S$; in this case, the part $S_{\varepsilon} \subset S$ together with the part $T_{\varepsilon}$ of the cone surface $\partial G_{\rho}^{\varepsilon}$ form a closed piecewise smooth surface $S_{\varepsilon} \cup T_{\varepsilon}$ (with the consistent direction of the outer normals) which is the boundary of a simply connected bounded domain. Represent the integral on the right-hand side of (4.8) as the sum of two integrals according to the representation $S=S_{\varepsilon} \cup\left(S \backslash S_{\varepsilon}\right)$. Since $\Psi_{\sigma}(y-x)$ is a regular solution of the Poisson equation, by Green's formula, the integral over the part $S_{\varepsilon}$ is equal to the integral over $T_{\varepsilon}$; moreover, $\Psi_{\sigma}(y-x)$ satisfies inequalities (4.7) and (4.9) for $y \in T_{\varepsilon}$ and $x \in M$, and the extended function $U(y)$ together with its gradient is bounded by a constant depending on $\varepsilon$. Therefore, the modulus of the integral over the part $S_{\varepsilon}$ does not exceed the quantity

$$
\frac{\text { const }}{1+\delta^{2} \sigma^{2}}, \quad \sigma \geq 0
$$

with a constant depending on $\rho, \varepsilon, \delta$, and the diameter of the domain $\Omega_{\rho}$. Since $|y| \geq \tau\left(y_{3}-\varepsilon\right)$, $y_{3} \geq \varepsilon$, when $y \in S \backslash S_{\varepsilon}$ and $x \in K$ and $f_{1}(y), f_{2}(y) \in C\left(S_{0}\right) \cap L(S)$, these inequalities remain valid for the modulus of the integral over $S \backslash S_{\varepsilon}$ (of course, with other constants). Hence, we have (4.10).

Sufficiency: Under the assumptions of the theorem, define functions $U(x), x \in G_{\rho} \backslash S_{0}$, by the right-hand side of (4.7). Consider the first term on the right-hand side of (4.7). Since $\Psi_{\sigma}(y)$ satisfies the Poisson equation in $G_{\rho}$ for $\sigma \geq 0$, the function $J(\sigma, x)$ satisfies the Poisson equation with respect to $x$ in $G_{\rho}$ for $\sigma \geq 0$. Therefore, we conclude from (4.10) that the first term on the right-hand side of (4.7) satisfies the Poisson equation in $G_{\rho}$ as the limit of the uniformly converging sequence of the solutions of the Poisson equations

$$
U_{n}(x)=\int_{0}^{n} J(\sigma, x) d \sigma, \quad n=1,2, \ldots
$$

The second and third terms are the potential difference of the volume, single, and double layers and represent one solution of the Poisson equation in $\Omega_{\rho}$ and another in $\Omega_{\rho}^{\prime}=G_{\rho} \backslash \bar{\Omega}_{\rho}$. Therefore, the right-hand side of (4.7) defines two different solutions of the Poisson equations $U^{+}(x)$ and $U^{-}(x)$ in $\Omega_{\rho}$ and $\Omega_{\rho}^{\prime}$. If $x^{1}$ and $x^{2}$ are two points on the normal at $x \in S_{0}$ symmetric with respect to $x$, then

$$
\lim _{x^{1} \rightarrow x}\left[U^{+}\left(x^{1}\right)-U^{-}\left(x^{2}\right)\right]=f_{1}(x), \quad \lim _{x^{1} \rightarrow x}\left[\frac{\partial U^{+}}{\partial n}\left(x^{1}\right)-\frac{\partial U^{-}}{\partial n}\left(x^{2}\right)\right]=f_{2}(x), \quad x \in S_{0} ;
$$


moreover, the limit relations hold uniformly in $x$ on each compact part $S_{0}$. If $\max y_{3}<x_{3}$, where $y \in S$ and $x \in G_{\rho}$, then $\operatorname{Re} w=y_{3}-x_{3}<0$ and $\Phi_{\sigma}(y-x)$ and its derivatives satisfy inequalities (4.6) and (4.3). Now, from formula (4.5), which is equivalent to (4.7), we see that $U^{-}(x)=0$ and $U^{-}(x) \equiv 0, x \in \Omega_{\rho}$, by the uniqueness theorem. It is clear that $U^{-}(x)$ extends smoothly to $\Omega_{\rho}^{\prime} \cup S_{0}$. Then $U^{+}(x)$ extends smoothly as a function of the class $C^{1}\left(\Omega_{\rho} \cup S_{0}\right)$ (see [29]). Consequently,

$$
U^{+}(x)=f_{1}(x), \quad \frac{\partial U^{+}}{\partial n}(x)=f_{2}(x), \quad x \in S_{0} .
$$

Now, we set $U(x)=U^{+}(x), x \in \Omega_{\rho} \cup S_{0}$. Theorem 3 is proved.

\section{REFERENCES}

1. Aizenberg L. A., Tarkhanov N. N. An abstract Carleman formula. Dokl. Math., 1988. Vol. 37, No. 1. P. $235-238$.

2. Aizenberg L. A. Carleman's Formulas in Complex Analysis. Theory and Applications. Math. Applications, vol. 244. Dordrecht: Springer, 1993. 299 p. DOI: 10.1007/978-94-011-1596-4

3. Alessandrini G., Rondi L., Rosset E., and Vessella S. The stability for the Cauchy problem for elliptic equations. Inverse problems, 2009. Vol. 25, No. 12. Art. no. 123004. DOI: 10.1088/0266-5611/25/12/123004

4. Arbuzov E. V., Bukhgeim A.L. The Carleman's formula for the Maxwell's equations on a plane. Sib. Elektron. Mat. Izv., 2008. No. 5. P. 448-455. (in Russian)

5. Belgacem F. B. Why is the Cauchy problem severely ill-posed? Inverse problems, 2007. Vol. 23, No. 2. P. 823-836. DOI: $10.1088 / 0266-5611 / 23 / 2 / 020$

6. Bers L., John F., Schechter M. Partial Differential Equations. NY: Interscience, 1964. 343 p.

7. Blum J. Numerical Simulation and Optimal Control in Plasma Physics. With Applications to Tokamaks. NY: John Wiley and Sons Inc., 1989. 363 p.

8. Carleman T. Les Founctions Quasi Analitiques. Gauthier-Villars, Paris, 1926. 115 p. (in French)

9. Chen G., Zhou J. Boundary Element Methods. London etc.: Academic Press, 1992. 646 p.

10. Colli-Franzone P., Guerri L., Tentoni S., Viganotti C., Baruffi S., Spaggiari S., Taccardi B. A mathematical procedure for solving the inverse potential problem of electrocardiography. analysis of the time-space accuracy from in vitro experimental data. Math. Biosci., 1985. Vol. 77, No. 1-2. P. 353-396. DOI: $10.1016 / 0025-5564(85) 90106-3$

11. Dzhrbashyan M. M. Integral'nye preobrazovaniya i predstavleniya funkcij v kompleksnoj oblasti [Integral Transformations and Representations of Functions in a Compkjoplex Domain.] M.: Nauka, 1966. 670 p. (in Russian)

12. Ermamatova Z. E. Carleman's formula of a solution of the Poisson equation. Int. J. Integrated Education, 2021. Vol. 4, No. 7. P. 112-117.

13. Fasino D., Inglese G. An inverse Robin problem for Laplace's equation: theoretical results and numerical methods. Inverse problems, 1999. Vol. 15, No. 1. P. 41-48. DOI: 10.1088/0266-5611/15/1/008

14. Fok V. A., Kuni F. M. On the introduction of a 'suppressing' function in dispersion relations. Dokl. Akad. Nauk SSSR, 1959. Vol. 127, No. 6. P. 1195-1198. (in Russian)

15. Gilbarg D., Trudinger N. S. Elliptic Partial Differential Equations of Second Order. Berlin-HeidelbergNY-Tokyo: Springer-Verlag, 1983. 513 p.

16. Goluzin G. M., Krylov V. I. A generalized Carleman formula and its application to analytic continuation of functions. Mat. Sb., 1933. Vol. 40, No. 2. P. 144-149. (in Russian)

17. Hadamard J. Lectures on Cauchy's Problem in Linear Partial Differential Equations. New Haven: Yale University Press, 1923. $334 \mathrm{p}$.

18. Hu X., Mu L., Ye X. A simple finite element method of the Cauchy problem for Poisson equation. Int. J. Numer. Anal. Model., 2017. Vol. 14, No. 4-5. P. 591-603.

19. Inglese G. An inverse problem in corrosion detection. Inverse problems, 1997. Vol. 13, No. 4. P. 977-994. DOI: $10.1088 / 0266-5611 / 13 / 4 / 006$ 
20. Ivanov V. K. Cauchy problem for the Laplace equation in an infinite strip. Differ. Uravn., 1965. Vol. 1, No. 1. P. 131-136. (in Russian)

21. Kabanikhin S. I. Inverse and Ill-Posed Problems: Theory and Applications. Berlin: De Gruyter, 2011. 459 p. DOI: $10.1515 / 9783110224016$

22. Lattès R., Lions J.-L. The Method of Quasi-Reversibility: Applications to Partial Differential Equations. Modern Analytic and Computational Methods in Science and Mathematics, vol. 18. NY: American Elsevier Pub. Co., 1969. 388 p.

23. Lavrentiev M. M. Some Improperly Posed Problems of Mathematical Physics. Springer, Berlin, 1967. 76 p. DOI: $10.1007 / 978-3-642-88210-4$

24. Lavrentiev M. M. On Cauchy problem for linear elliptical equations of the second order. Dokl. Akad. Nauk SSSR, 1957. Vol. 112, No. 2. P. 195-197. (in Russian)

25. Makhmudov O.I. The Cauchy problem for a system of equations of the theory of elasticity and thermoelasticity in space. Russian Math. (Iz. VUZ), 2004. Vol. 48, No. 2. P. 40-50.

26. Makhmudov O., Niyozov I., Tarkhanov N. The Cauchy problem of couple-stress elasticity. Contemporary Math., 2008. Vol. 455. P. 297-310.

27. Makhmudov K. O., Makhmudov O. I., Tarkhanov N. Equations of Maxwell type. J. Math. Anal. App., 2011. Vol. 378, No. 1. P. 64-75. DOI: 10.1016/j.jmaa.2011.01.012

28. Mergelyan S. N. Harmonic approximation and approximate solution of the Cauchy problem for the Laplace equation. Uspekhi Mat. Nauk, 1956. Vol 11, No. 5. P. 3-26.

29. Miranda C. Partial Differential Equations of Elliptic Type. Berlin, Heidelberg, NY: Springer, 1970.370 p. DOI: $10.1007 / 978-3-642-87773-5$

30. Peicheva A. S. Regularization of the Cauchy problem for elliptic operators. J. Sibirian Federal University. Math. Phy., 2018. Vol. 2. P. 191-193. DOI: 10.17516/1997-1397-2018-18-2-191-193

31. Sattorov E. N. Regularization of the solution of the Cauchy problem for the generalized MoisilTheodoresco system. Diff. Equat., 2008. Vol. 44. P. 1136-1146. DOI: 10.1134/S0012266108080119

32. Sattorov E. N. Continuation of a solution to a homogeneous system of Maxwell equations. Russian Math. (Iz. VUZ), 2008. Vol. 52, No. 8. P. 65-69. DOI: 10.3103/S1066369X08080094

33. Sattorov E. N. On the continuation of the solutions of a generalized Cauchy-Riemann system in space. Math. Notes, 2009. Vol. 85, No. 5. P. 733-745. DOI: 10.1134/S0001434609050149

34. Sattorov E.N. Regularization of the solution of the Cauchy problem for the system of Maxwell equations in an unbounded domain. Math. Notes, 2009. Vol. 86, No. 6. P. $422-431$. DOI: 10.1134/S0001434609090156

35. Sattorov E. N. Reconstruction of solutions to a generalized Moisil-Teodorescu system in a spatial domain from their values on a part of the boundary. Russian Math., 2011. Vol. 55, No. 1. P. $62-73$. DOI: 10.3103/S1066369X11010075

36. Sattorov E. N., Ermamatova Z. E. Recovery of solutions to homogeneous system of Maxwell's equations with prescribed values on a part of the boundary of domain. Russian Math., 2019. Vol. 63, No. 2. P. 35-43. DOI: 10.3103/S1066369X19020051

37. Sattorov E. N., Ermamatova F. E. Carleman's formula for solutions of the generalized Cauchy-Riemann system in multidimensional spatial domain. Contem. Problems in Mathematics and Physics, 2019. Vol. 65, No. 1. P. 95-108. DOI: 10.22363/2413-3639-2019-65-1-95-108

38. Sattorov E. N., Ermamatova F. E. On continuation of solutions of generalized Cauchy-Riemann system in an unbounded subdomain of multidimensional space. Russian Math., 2021. Vol. 65, No. 2. P. 22-38. DOI: $10.3103 /$ S1066369X21020031

39. Sattorov E.N., Ermamatova F.E. Cauchy problem for a generalized Cauchy-Riemann system in a multidimensional bounded spatial domain. Diff. Equat., 2021. Vol. 57, No. 1. P. 86-99. DOI: 10.1134/S0012266121010079

40. Sattorov E. N., Mardanov D. A. The Cauchy problem for the system of Maxwell equations. Siberian Math. J., 2003. Vol. 44, No. 4. P. 671-679. DOI: 10.1023/A:1024788607969

41. Tarkhanov N. N. The Carleman matrix for elliptic systems. Dokl. Akad. Nauk SSSR, 1985. Vol. 284, No. 2. P. 294-297. (in Russian)

42. Tarkhanov N. N. The Cauchy Problem for Solutions of Elliptic Equations. Berlin: Akad. Verl., 1995. $479 \mathrm{p}$. 
43. Tikhonov A. N. On the solution of ill-posed problems and the method of regularization. Dokl. Akad. Nauk SSSR, 1963. Vol. 151, No. 3. P. 501-504. (in Russian)

44. Tikhonov A. N., Arsenin V. Y. Solutions of Ill-Posed Problems. NY: John Wiley \& Sons, 1977.

45. Yarmukhamedov Sh. On the Cauchy problem for Laplace's equation. Dokl. Akad. Nauk SSSR, 1977. Vol. 235, No. 2. P. 281-283. (in Russian)

46. Yarmukhamedov Sh. Continuing solutions to the Helmholtz equation. Doklady Math., (1997). Vol. 56 , No. 3. P. 887-890.

47. Yarmukhamedov Sh. A Carleman function and the Cauchy problem for the Laplace equation. Sib. Math. J., 2004. Vol. 45, No. 3. P. 580-595 DOI: 10.1023/B:SIMJ.0000028622.69605.c0

48. Yarmukhamedov Sh. Representation of Harmonic Functions as Potentials and the Cauchy Problem. Math. Notes, 2008. Vol. 83, No. 5. P. 693-706. DOI: 10.1134/S0001434608050131 